\title{
Quantum Gravity Sensor by Curvature Energy: their Encoding and Computational Models*
}

\author{
Francisco Bulnes, $\mathrm{PhD}$, PostDoct \\ Head of Research Department in Mathematics and Engineering \\ Technological Institute of High Studies of Chalco \\ Chalco, P. C. 56641, State of Mexico, Mexico
}

\begin{abstract}
Through of the concept of curvature energy encoded in non-harmonic signals due to the effect that characterizes the curvature as a deformation of field in the corresponding resonance space ( and an obstruction to the displacement to the corresponding shape operator) is developed and designed a sensor of quantum gravity considering the quantized version of curvature as observable of gravitational field where the space is distorted by the strong interactions between particles, interpreting their observable in this case, as light fields deformations obtained on space-time background. To the application of this measurement we use a hypothetical particle graviton modeled as a magnetic dilaton which must be gauge graviton (gauge boson). Also are obtained several computational models of these photonic measurements, likewise their prototype photonic devices.
\end{abstract}

Keywords-Curvature energy; magnetic dilaton; quantum gravity sensor; strong interactions; quantum computing

\section{INTRODUCTION}

The gravitation is the study of engendered field for the presence of matter in the Universe. This study begins with the determination of a field $\boldsymbol{G}$, and their sections $G_{i j}(i, j=1,2,3, \ldots, N)$, , in the space through the universal gravitation law applied globally and locally, this last, considering the earthy ambit where is computed the acceleration in free fall of all bodies that fall in the event horizon of the Earth.

Subsequently and when the inertial effects due to the field and considering their coordinates system, likewise their transformations are established the relativistic models that consider chart systems (inertial reference systems of Galileo Galilei) to the determination of the field and their effects in said reference frame.

The study initiated with the universal gravitation is re-taken by Einstein in the frame of the curved geometry defining a geometrical stage for the only presence of the gravitational field $\boldsymbol{G}$, , defined this stage for the curvature, taking the Einstein field equations to symmetric metric tensor :

$$
R^{\alpha \beta}-\frac{1}{2} R g^{\alpha \beta}=-\chi T^{\alpha \beta},
$$

TESCHA (Technological Institute of High Studies of Chalco) and COMECYT(State of Mexico Council of Science and Technology). being $R^{\alpha \beta}$, the curvature tensor or Riemannian tensor of the space-time. The constant $\chi$, is the gravitation constant given by Einstein equal to $8 \pi G / c^{2}$.

We can simplify the field equations as

$$
R+\chi T=0
$$

which establishes that the space-time experiments $a$ curvature in presence of energy. That is to say

$$
R=-\chi T,
$$

The negative sign in $-\chi T$, is a convention, but can serve to remember us that the energy curves to the space-time to inside.

Then as is had said the gravity "winging" to the things to their source. A little change in the paradigm here is that the mass not is attracted between these "wing" on the space-time. These, curve to the space-time.

One important observation is that when is found with $\chi T$, must be imagined a spherical density. In change, when we find with $R$, the curvature is of the space-time as deformation of the spatial stage due the presence of matter. The exact values not are important in this last description.

In the non-Euclidean space-time or Riemannian space the symmetric tensor of the metric is $O^{\mu v(S)}$. This is the same that " $g$ " the metric tensor but referred to distances where distance is symmetric as functional. Here the super fix " $S$ " means symmetric.

The asymmetric tensor of the more general metric (which could consider electromagnetic fields as field gauges to measurements of field) is defined through the external or tensor product between tetrads (four metric vectors), that is to say:

$$
o_{\mu v}^{a b}=o_{\mu}^{a} o_{v}^{b}=o_{\mu v}^{a b^{(S)}}+o_{\mu v}^{a b^{(A)}},
$$

Asymmetric form haves inside herself as well as symmetry and anti-symmetry. The anti-symmetric component from (3) is $o_{\mu v}^{a b^{(A)}}$. The anti-symmetric tensor of the metric is defined through the wedge product of two tetrads:

$$
o_{\mu v}^{a b^{(A)}}=o_{\mu}^{a} \wedge o_{v}^{b}
$$


The action of the product of the tensors of curvature $R^{\alpha \beta}$ and $o_{\mu}^{\alpha}$, will establish an action of "torsing the action of gravity", which can be measurable as distortions produced from the gravity.

Then with the appearing of the quantum mechanics, more specifically, the QFT, and their interrelation with the gravitation is searched establish the cause of the field through the quantum interactions that generate this.

Then in this new "exhibition of gravity" the Einstein field equations (1) can be re-written as:

$$
R_{\mu}^{a}-\frac{1}{2} R o_{\mu}^{a}=\chi T_{\mu}^{a}
$$

and using the fact that now our new metric tensor can be expressed as:

$$
g_{\mu v}^{a b(A)}=o_{\mu}^{a} \wedge o_{v}^{b}
$$

we arrive to the new field equation to electrodynamics that is generally covariant:

$$
o_{\mu}^{a} \wedge\left(R_{v}^{b}-\frac{1}{2} R o_{v}^{b}\right)=\chi o_{\mu}^{a} \wedge T_{v}^{b},
$$

which give us the spin or torsion of the field.

In this asymmetric space-time model are obtained field models that reflect this torsion. For example the Asymmetric field theory given by Yang-Mills where this theory provides an extension of Maxwell theory to the case of non-Abelian fields. In this dimensions raise the wrappings and the loop contributions that will contribute to the energy micro-states used to define electromagnetic signal effects of power that can consigned in a harmonic analyzer.

Subsequently with the string theory [1] and wanting to solve the problem of the super-symmetry, is developed the concept of quantum gravity which establish through of hypothetical particle called "graviton" [2] (which by the duality principle of the $Q F T$, on field/particle) could establish the origin of the field $\boldsymbol{G}$, as the bosonic source of the gravitation.

After and under studies of the $Q E D$, are established indirect methods to the determination and detection of quantum gravity using gauge fields or gauge bosons respect to the background radiation of the Universe and these produce for "backreaction" a measure of the distortion that produce the presence of the field $\boldsymbol{G}$, as the causing of the variation and distortion of the gauges field with the microwaves of the spacetime.

Then these variations can be the pattern that the Cartan tetrads $o_{\mu \nu}^{a b}$, define to realize the measurements of quantum gravity fluctuations and that can be the solutions to one obtained quantum field equation, analogous to the Dirac equation considering the electromagnetic and gravitational fields ${ }^{2}$ together and whose solutions can be measurable gravitational waves in electromagnetic waves through of filters of signals:

$$
(\square+\chi T) o_{\mu}^{a}=0,
$$

\section{H-FiELDS IN A GENERALIZED CURVATURE TENSOR AND FIRST BOSON-FERMION MEASUREMENTS}

Considering the integration formalism through the total action integral of $g_{\mu v}^{a b(A)}$, that comes from the sum of the partial actions due to the curvature tensor and of the electromagnetic tensor include in this last the fermion self interactions induced by quantum torsion we have that a second integral of (7) is:

$$
\begin{aligned}
& \mathfrak{I}_{\text {TOTAL }}=\frac{1}{2 \kappa} \int d^{4} \operatorname{XOO}_{\mu}^{a} o_{v}^{b} R(\varpi)+\frac{i}{2} \int d^{4} x O \times \\
& \times\left(o_{\mu}^{a}\left(\psi \gamma^{a} \mathcal{D}_{\mu}(\varpi, A) \psi-\overline{\mathcal{D}_{\mu}(\varpi, A) \psi} \gamma^{a} \psi\right)\right)- \\
& -\int d^{4} x o \frac{3}{10} \kappa J_{(A)}^{\mu} J_{(A) \mu},
\end{aligned}
$$

Finally this global action defined in (9) can be re-written to fermions in gravity with torsion $[3,4]$, with a specific kind of torsion (Kalb-Ramond field strength [5]) inspirited from the string theory mentioned before ( $U V$ complete) can do the job of providing a constant, axial background in a local frame of $F R W-$ cosmology. Then the additional fermion-piece of the form

$$
\begin{aligned}
& \mathfrak{I}=\frac{\alpha}{2} \int d^{4} x o\left(\psi \gamma^{a} \mathcal{D}_{\mu}(\varpi, A) \psi-\overline{\mathcal{D}_{\mu}(\varpi, A) \psi} \gamma^{a} \psi\right), \\
& \alpha=c t e
\end{aligned}
$$

together with Dirac kinetic terms, the fermion action reads:

$$
\begin{aligned}
& \mathfrak{J}_{\text {Dirac-Holst-Fermi }}=\frac{\alpha}{2} \int d^{4} x o\left(\psi \gamma^{a}\left(1-i \alpha \gamma_{5}\right) \mathcal{D}_{\mu} \psi-\right. \\
& \left.\overline{\mathcal{D}}_{\mu} \psi \gamma^{a}\left(1-i \alpha \gamma_{5}\right) \psi\right),
\end{aligned}
$$

where inside the integrand are involved the Dirac equations to the differentiating fermions in the non-Harmonic analysis

${ }^{2}$ Gravity with torsión contains anti-symmetric parts in the connection:

$$
\omega_{\mu}^{\alpha b}=\varpi_{\mu}^{a b}+K_{\mu}^{a b},
$$

where $\varpi_{\mu}^{\alpha b}=o_{\mu}^{a} \partial_{\mu} o^{v b}+o_{\nu}^{a} o^{\sigma b} \Gamma_{\sigma \mu}^{v}=o_{\nu}^{a} o_{; \mu}^{v b}$. Then we have a co-torsion tensor $K_{\mu}^{\alpha b}=o_{\nu}^{a} o_{\rho}^{b} K_{v \rho}^{a b}$, such that $K_{\mu}^{v \rho}=-K_{\mu}^{\rho v}$.

\footnotetext{
${ }^{1}$ That is to say, the new metric tensor is anti-symmetric.
} 
that appear in the anti-symmetric behavior of the curvature field measured for quantum interactions (see Figure 1).

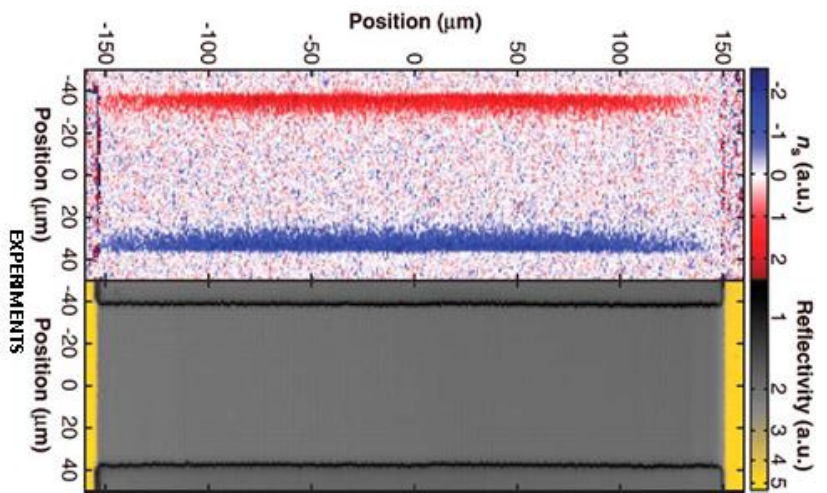

Fig.1. Differentiating fermions in the non-Harmonic analysis that appear in the anti-symmetric behavior of the curvature field measured for quantum interactions.

Inside of the microscopic UV complete underlying theory of quantum gravity if we consider the massless gravitational multiplet of "closed" strings such as spin 0 , scalar or dilaton, spin 2 , traceless symmetric rank 2 tensor or the graviton, and spin 1, antisymmetric rank 2 tensor or electromagnetic tensor, with the Kalb-Ramond field $B_{\mu \nu}=-B_{v \mu}$, we can have a gauge invariant to effective field theories (in low energy scale $E<<M_{s}$ ) given for $B_{\mu v} \rightarrow B_{\mu v}+\partial_{[\mu} \theta(x)_{v]}$, which depend only on the field strength $H_{\mu v \rho}=\partial_{[\mu} B_{v \rho]}$. Then we give the Bianchi identity:

$$
\partial_{[\sigma} H_{\mu v \rho]}=0 \text {, }
$$

But to the detected anomalies through the gravitational versus gauge interactions cancellations of strings (necessaries to the apperceiving of the gravitational waves letting only the gravitational strings) is required a re-definition of the $\mathrm{H-}$ fields given in (12) using the extension due to the Majorana neutrinos masses from (three loop) anomalous terms with axion-neutrino couplings. Then the corresponding extended Bianchi identity to these anomalous terms come given as:

$$
H_{\mu v \rho}=\partial_{[\mu} B_{v \rho]}+\frac{\alpha^{\prime}}{2 \kappa}\left(\Omega_{L}-\Omega_{V}\right),
$$

where result interesting study the phase-space density derived from the difference between of the Chern-Simons three forms [6] $\Omega_{L}$, and $\Omega_{V}$, where as Lorentz Chern-Simons three form $\Omega_{L}$, is considered the corresponding to neutrinos, and to the case of the gauge Chern-Simons three form is considered the corresponding electro-gravitational formalism in gauge theory.

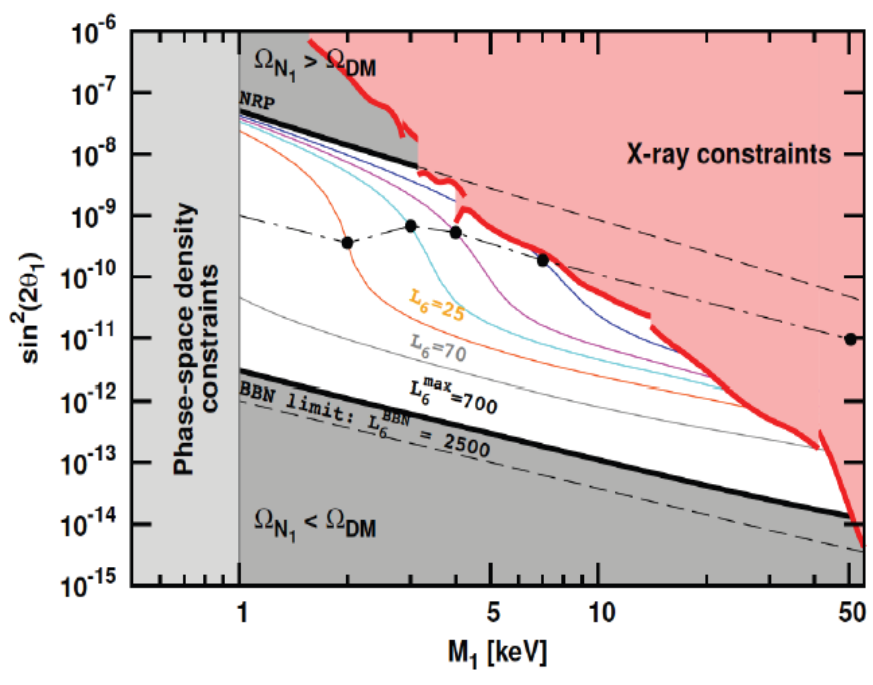

Fig.2. Leptogenesis/Baryogenesis model consistent with BBN- structure formation data in the Universe and all other astrophysical constraints [7].

A theoretical study related to the propagation of photons in quantum gravity, shows that the region of space- time of the mechanical well of a singularity is supported by an energy that decreases asymptotically in the infinite. This hypothetical energy we can construct it with the expression of a Lagrangian like the given in (Table 2 [8-10]) with cylindrical gravitational wave by the dilaton (gauge particle):

$\Phi=\left(1 / 10000\left(\exp (-4 \xi) J_{v, x}(3 \xi, 1)+\exp (-4 \xi) Y_{v, x}(2 \xi, 1)\right),(12)\right.$ wave model for energy of gravitational waves (see last line of the Table 3)) [8]. Also see the figure 3 [8].

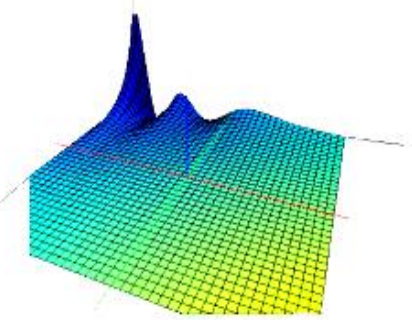

Fig.3. Gravitational alteration perceive by the censor designed by (13) when is obtained a great alteration of energy near the singularity of the space-time.

\section{Curvature Energyto Design A Quantum Gravity SENSOR}

According to our curvature studies that come from a theoretical sensor of curvature in presence of the incurve region of the space and detected by a light wave $[8,11]$, the curvature follows being the more important geometrical invariant that appear as a geometrical effect in the space when appears a variation of the field due to the deformations of the light geodesics encoding as light waves that transit near of material sources of space where

The data of these encodings where have been consigned in a first model of "gravitational waves" suggest that the detection 
of gravity can be through of the energy obtained by a cosmic censorship, given by

$$
[\log \phi(\xi(s))]^{2}\left[\int \log \sigma(s)\right]^{2} \geq\left(\int \Omega\left(1-\nabla^{2} \log \Omega\right)\right)^{2} \geq 4 \pi \int \Omega,
$$

This is a Hilbert inequality that through "curvature energy" measured with models of Gaussian and normal curvatures establishes an energy range where the curvature energy can exist if their Lagrangian haves a Maxwellian component. Observe the certain bound of roundness obtained through of the implementation of a spherical operator $[9,12]$, we can use one particular case given by measurement for light waves contemplated in the Bulnes's theorem published in ASME 2009, [11].

The possible integral expression (13) of the total Hamiltonian of electromagnetic energy establishes a condition of domineering energy [11], when there exists curvature in the 3-dimensional space (that is to say, if the energy is given by this roundness censor, there is curvature measured like energy that makes the censoring appear and measured directly using positioned of the sensor device). This makes possible to obtain a curve of perception of curvature that measures the condition of domineering energy in the sensor to establish their position in the surface [12] and of this way to measure in every point of the surface their curvature.

But the cosmic censorship, which due to their design through classical electromagnetic fields, requires of a supermassive source to obtain a significant variation of the gravity presence (see the figure 4).

The gravitational waves can be modeled by non-harmonic signals produced by detection of the super-massive source like a black hole in the Universe [8].
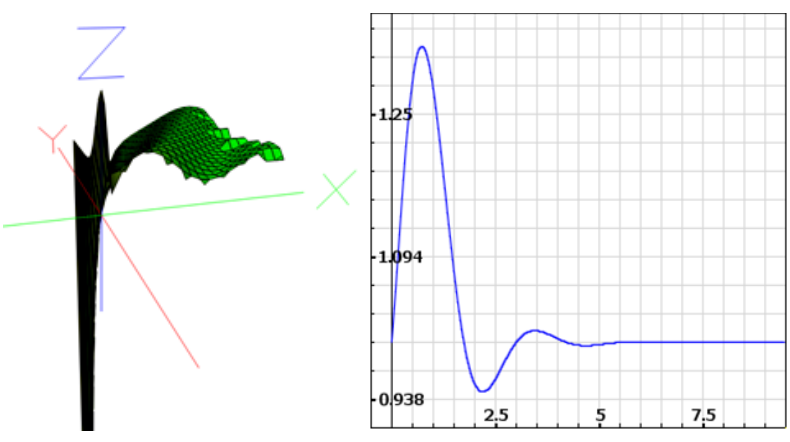

Fig.4.

Then using the difference of energy that can detect a sensor of gravity that re-collect little variations or fluctuations due to inertial effects of the matter existence in the space-time, we can measure curvature we base in the idea of consignee in angles from the resonances [11]. Such is the case, for example, the use of an inertial device as an accelerometer which with a capacity of dynamic perception given by their electrostatic force $F_{e}=1 / 2 A V^{2} / d^{2}$, whose deflection is consigned in a $g-$ cell component.

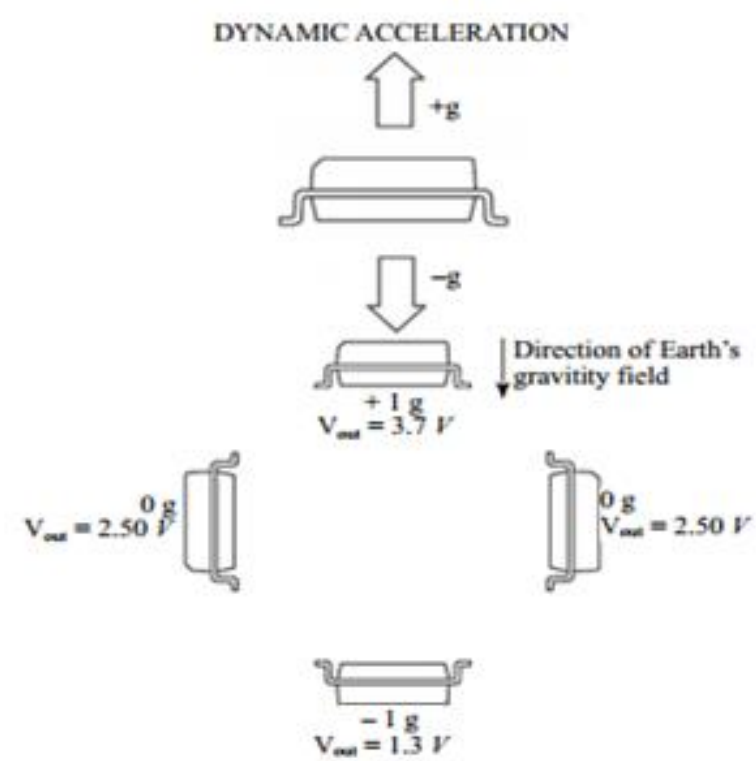

Fig.5. Accelerometer type model used to perceive variations of gravity in the usual sense [12]. This will be modified to can receive the fine variations or fluctuations between boson gauges and microwaves in background. Their gravity element will substitute by non-Newtonian fluid which wills consignee the little variations of gravity from the values of each parasite capacitors.

This force is designed in our sensor by the basic isotopic component of Gaussian factor to lectures of curvature defined as $\alpha(-1)^{2} \bullet 1 \bullet\left(4 \pi^{2}\right)$, where $(-1)^{2}$, is the basic charge given in function of the milimetric potential $V$, the factor $A=4 \pi^{2}$, that there is in the surface of the sphere of radius the unit $S^{2}(1), \alpha$, is the degree of the spherical map used in the transduction of the physical model to measure, which comes like a factor of electromagnetic adjustment of the sensor on the curved surface and the factor 1, is the positive charge generated inside the sensor (see figure 5). This is equivalent to the product of the force for the square of the distance of separation between the plates sensors of the nucleus of the sensor, where one gathers the change of the load according to the surface (negative load), and other gathers the charge invested in the process of detection (positive charge). The resultant deflection is measured by the accelerometer's control ASIC and a proportional output voltage results $[12,13]$. This procedure assures that both the mechanical ( $g-$ cell) and electronic sections of the accelerometer are functioning [14].

How measure curvature of the space-time from the concept of quantum gravity interpreting their observable in this case, as light field deformations obtained on space-time background?

Extra-poling the idea of the cosmic censorship to the quantum field fluctuations due to interacting of a dilaton with the microwave background we can detect through the difference scattering of fermions detected in each case; particles/anti-particles. In this case are neutrinos/anti-neutrinos where the curvature will be given for the sufficient quantity of sterile neutrinos that must appear to reproduce observed oscillations which express induced torsion. The apparent 
flatness in the space is conserved and only in macroscopic region of the space-time is

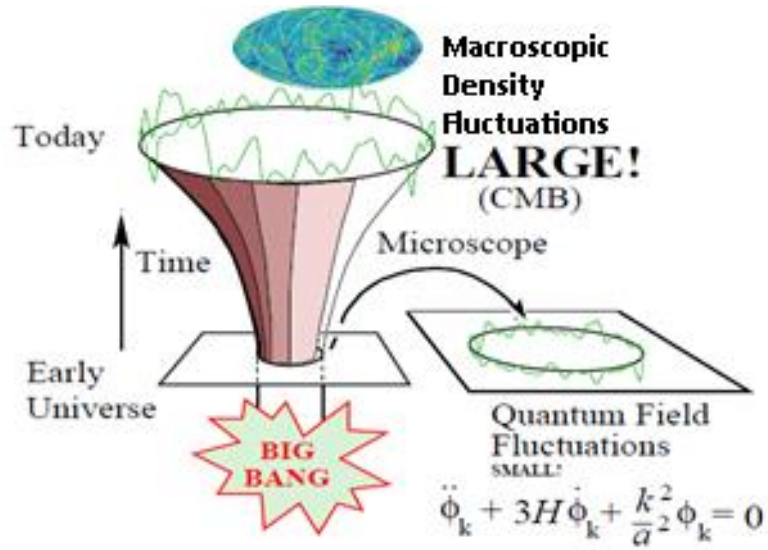

Fig.6.

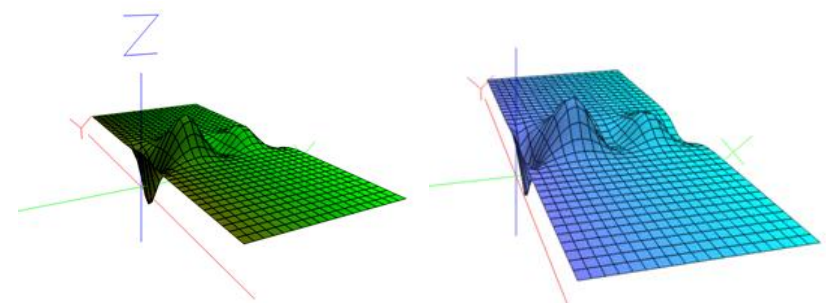

Fig.7. Modeling and measurements obtained by a theoretical sensor of quantum gravity designed accords with the quantum field fluctuations in the flatness of the space-time supported by the neutrinos/anti-neutrinos totality.

Using the concept of curvature energy mentioned in our introduction we can annunciate the following result which was published in [12]:

Theorem (F. Bulnes). III. 1. [12] Let $\mathfrak{a}$, be an accelerometer given by the device $D$, defined for their $g-$ cell whose curvature energy to the case 2 -dimensional is the bounded by the integral $\Lambda \int_{C} k^{2} d s,[11,15]$, where $\Lambda$, is an output electromagnetic factor from $D$, (to obtain curvature). Let $\mathbf{S}$, the corresponding shape operator on $S^{2}$ (and their contours), that is to say, is that whose normal curvature is given for $k(\mathbf{u})=\mathbf{u} \mathbf{S}_{p}(\mathbf{u})=\mathbf{u}(-1 / r) \mathbf{u}=-1 / r$. Then our curvature is defined for the inequality of voltage-curvature energy given by (1) whose energy is $\mathcal{E}_{k}=(1 / 2) V^{2} A \sin ^{2} \theta$.

\section{Proof. [12].}

Difference of Energy can be consigned in the quantum distortion given by the link-wave between graviton modeled as dilaton (gauged graviton) and the trace on relativistic Feynman diagram followed in quantum gravity. The quantum curvature as a different times in the causality and conformally in the space-time. The different deviations to the world-lines in each case show the curvature: spinor frame $[16,17]$

\section{PHOTONIC MEASUREMENTS AND COMPUTATIONAL MODELS}

Newly considering the effective gravitational action in string low-energy in terms a generalized curvature Riemannian tensor, where the Christoffel connection includes the $\mathrm{H}$ fields, that is to say, $\Gamma_{v \rho}^{\mu}=\Gamma_{v \rho}^{\mu}+\frac{\kappa}{\sqrt{3}} H_{v \rho}^{\mu} \neq \Gamma_{\rho v}^{\mu}$, defined in (13), we can give the $4-$ dimensional action:

$$
\begin{aligned}
& S^{(4)}=\int d^{4} x \sqrt{-g}\left(\frac{1}{2 \kappa^{2}} R-\frac{1}{6} H_{\mu \nu \rho} H^{\mu \nu \rho}\right) \\
& =\int d^{4} x \sqrt{-g}\left(\frac{1}{2 \kappa^{2}} R-\frac{1}{3} \kappa^{2} H_{\mu \nu \rho} H^{\mu \nu \rho}\right),
\end{aligned}
$$

where the dual of $H$, in four dimensions comes given by the differential equation:

$$
-3 \sqrt{2} \partial_{\sigma} b=\sqrt{-g} \in_{\mu v \rho \sigma} H^{\mu v \rho},
$$

where $b(x)$, is a pseudo-scalar that defines the KalbRamond axion. Then to a dilaton $\Phi$, that satisfies (15) having the properties described in (8) as gravitational wave, we have the differential equation:

$$
H^{\mu v \rho}=o^{2 \Phi} \in_{\mu v \rho \sigma} \partial^{\sigma} b(x),
$$

The linear dilaton solution in string frame, (or logarithmic in FRW time in Einstein frame) with conformally flat Einsteinframe target space-time is exact in all orders of a parameter , that appears in (11).

Using the principles dictated in (9)-(11) and the differentiating fermions in the non-Harmonic analysis that appear in the anti-symmetric behavior of the curvature field measured by quantum interactions we can give the following action that comes from of the Majorana states in fermionic field theories with $H$-torsion:

$$
S_{\psi}=-\frac{3}{4} \int d^{4} \sqrt{-g} S_{\mu} \psi \gamma^{\mu} \gamma^{5} \psi,
$$

Using the extra-charge created by the fermion interaction (central charge of underlying world-sheet conformal field theory [16]) where

$$
b(x)=\sqrt{2} e^{-\phi_{0}} \sqrt{Q^{2}} \frac{M_{s}}{\sqrt{n}} t, \forall n \in \mathbb{Z},
$$

and using said charge to create a basic charge in a component of $g$ - cell, and also using our theorem on curvature considering an isotopic component of Gaussian factor to lectures of curvature we can define a sensor whose 3ball of non-Newtonian fluid can receive these signal and reinterpreting through voltage-curvature energy, such and say the theorem III. 1, these data as little electrical tensions that come from the surface of the 3-ball which can be sensored as little 
change in the background due to the dilaton in interaction with this.

An experiment is done with a little accelerometer that include a charged ball whose charge vary in the time when this accelerometer change their position respect to their horizontal frame (defined this for their Ecuador) given in their $g$-cell change (see the figure 8). We use two leds to establish the polarization effect created in natural form by the fermionic behavior.

The before can to help define a classical accelerometer in the earth's gravity. The curvature will be able to express itself like a Gaussian curvature according to spherical harmonics given by Legendre polynomials.

The sensor is a sensor of free fall that can register different force factors $G$. The difference is consigned by the Hall effect obtained by the difference scattering of fermions detected in each case; particles/anti-particles. The actions of change can be reprogrammed by the proper device considering these to be a Lagrangian action given for [8].

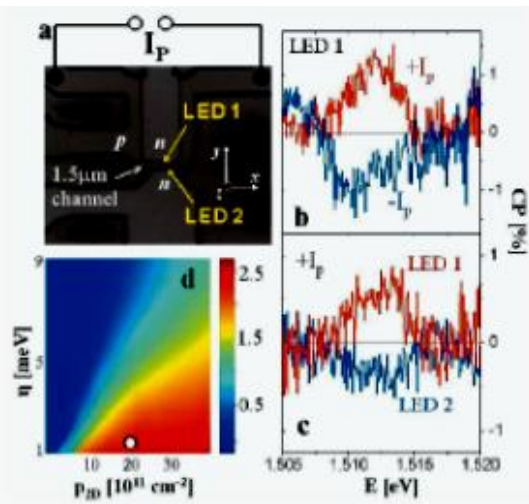

\section{SENSOR ON S 3 :}

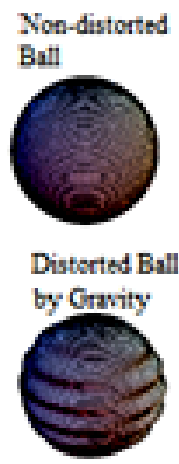

Fig.8. The difference is consigned by the Hall effect obtained by the difference scattering of fermions detected in each case; particles/anti-particles.

Then extrapolating this experiment in the ambit of the photonics the folds or "creases" in a deformable sphere are oscillations in the Universe which comes given by the mixture of neutrinos/antineutrinos for the eco of the Early Universe which maintained their basic non-spherical symmetry until our actual Universe and which can be expressed through of their Lagrangian as:

$$
\mathcal{L}=\mathcal{L}_{f}+\mathcal{L}_{I}=\sqrt{-g} \psi\left[\left(i \gamma^{a} \partial_{a}-m\right)+\gamma^{a} \gamma^{5} B_{a}\right] \psi,
$$

where $\Psi$, and $\psi$, are component of the spinor $\Psi$. The oscillations are received as spherical auto-modes of the alteration of central charge $Q$, obtained by the differentiated fermionic process (see the figure 9) (extension of the model the axion $b(x)$ using total derivatives of the gravitational $c R^{\mu v \rho \sigma} \widetilde{R}_{\mu v \rho \sigma}$, and electromagnetic $c F^{\mu v} \widetilde{F}_{\mu v}$, [18] terms of the fields $o_{\mu}^{a}$, translated to $H-$ fields). a)
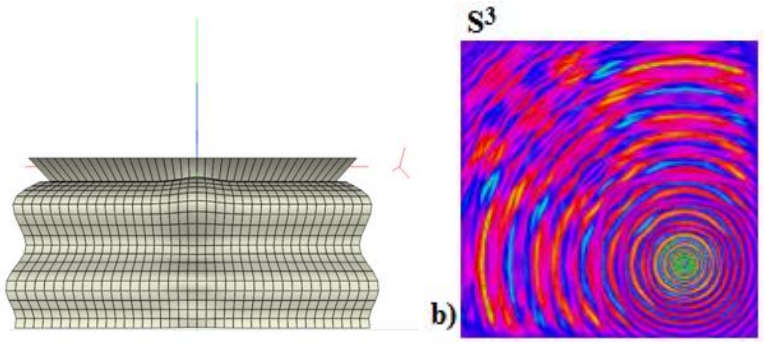

Fig.9. a). Comparison between the theoretical norm obtained by the connection given by (6) (whose distance formula) is (red curve) and WMAPSatellite measurements (black and blue curve) b). Measuring the presence of cosmic microwave background $(C M B)$ on the theoretical twistor surface of hypothetical ball $S^{3}$, in our sensor device. C). Image obtained by WMAP satellite [19].

The neutrinos/anti-neutrinos conform the asymmetry around black holes or singularities. Inside of singularities the gravitational field is dementia. Then their particle/anti-particle can be generated from the torsion. Using plane wave approximation is obtained different dispersion relations between particles/anti-particles at finite densities assuming constant background torsion (see the figure 10).
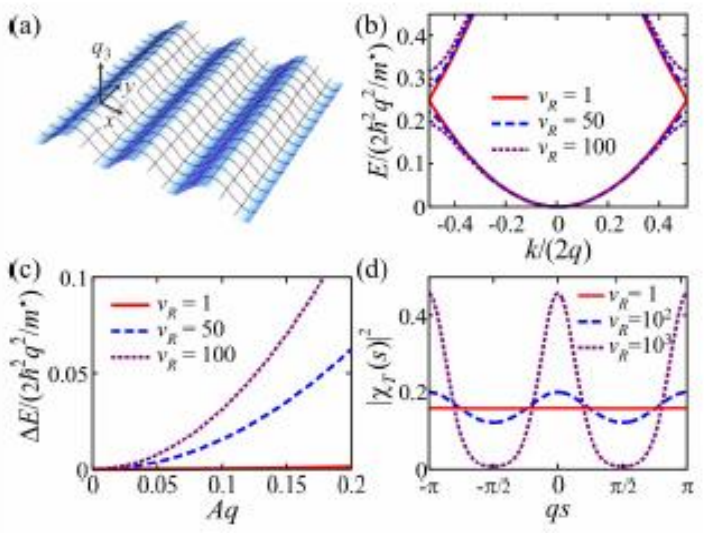

Fig.10. Quantized curvature energy in the harmonic case. a) The gravitational waves model is symmetric and obeys to the spherical symmetry. b), c) and d) Dispersion relations between particles/anti-particles.

Finally through a magnetic dilaton $\Phi$, we can give a model of magnetic distortion, that is to say, the energy curvature in the gravitational media can be translated as magnetic deformation of 4-dimensional part of the string of background radiation (see the figure 11).
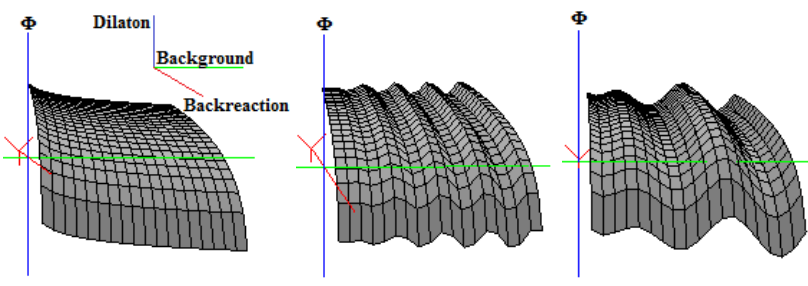

Fig.11. Dilaton measuring distortion due to quantum gravity, according to the model computacional magnetic $\Phi=\mathrm{y} \wedge \operatorname{sqrt}(\mathrm{y}+1)-((1 / 2) \log (\mathrm{x}+1 / \mathrm{x}+(\mathrm{x}-$ $1 / \mathrm{x})) \cos \theta$ ) (see equation (19)). The surface in a), represents the space-time area before the photons back-reaction with background radiation, their magnetic model is $\Phi=\mathrm{y} \wedge \operatorname{sqrt}(\mathrm{y}+1)-((1 / 2) \log (\mathrm{x}+1 / \mathrm{x}+(\mathrm{x}-1 / \mathrm{x})))$. In the surface $\mathrm{b})$, the distortion is measured by the magnetic oscillations $\cos \theta$, that is to say, the term 
of tough deviation $\theta$, in the figure 1 . In c), the distortion is attenuated by the background increasing the undulations $\left(\cos m \theta, \forall m \in \mathbb{Z}^{+}\right)$and increasing their amplitudes being not detectable for being under the background (green line).

The gravitational energy is the curvature energy obtained through components Bessel functions or polynomials (see the figure 12).

A)

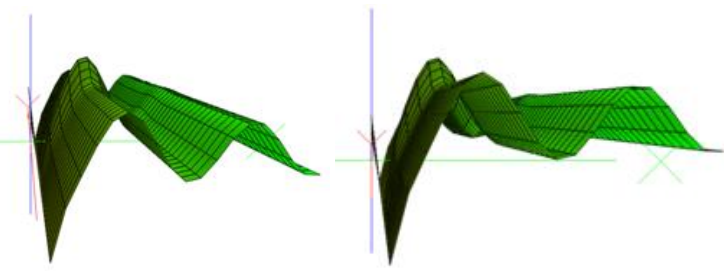

B)

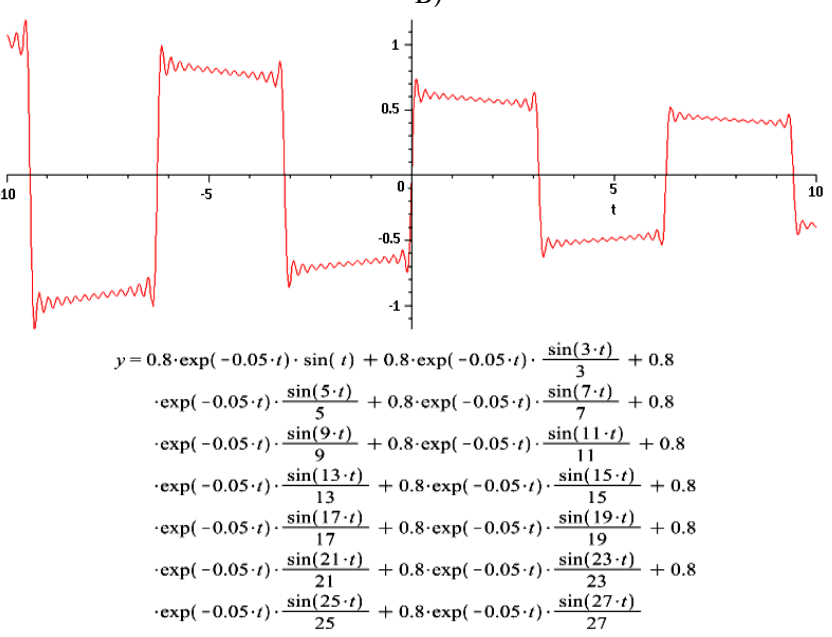

Fig.12. A) Curvature energy surfaces. The curvature represents an obstacle of the field flow and the "work" realized by the field to move their flow is translated in energy of curvature. B) Rescaling of the signal we can obtain a Bessel spectra corresponding to the energy curvature. The numerical operative sequences are realized to obtain this spectrum $[20,21]$.

\section{CONCLUSIONS}

The obtaining of geometrical models of quantum gravity comes to show the quantum behavior of observable obtained for photons that act on the background radiation (or microwave radiation) such as the torsion induced for $H$ - fields that through dilatons or gauge bosons can exhibit observables of gravitational field as curvature to quantum gravity, where the curvature in this last case is detected and viewed as curvature energy or gravitational energy that exists until our days as a eco of the times of the creating of the gravity in the step of a Early Universe.

Then the observational confirmation due to the gravitational background generating CPT violating effects in the Early Universe define experimentally by the particles/antiparticles differences in dispersion which implies CPTV effects of different space-time-curvature/spin couplings to neutrinos/anti-neutrinos [22].
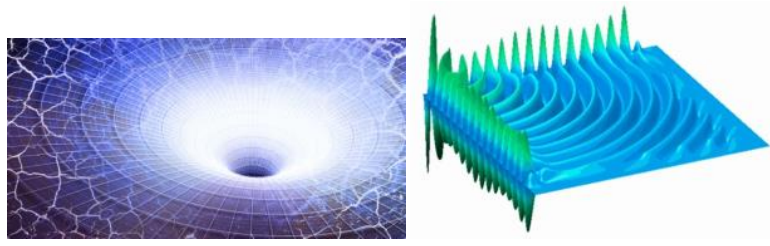

Fig.13. Gravitational alteration perceive by the quantum gravity sensor designed by the charge alteration in the fermion couplings. The waves that are spinors can be consigned in oscillations in the space-time-curvature/spin. The modeling of spinor "quantum waves" was done in ANSYS.

The curvature coupling to fermion spin (see the figure 14) may lead to different dispersion relations between neutrinos and anti-neutrinos (assumed dominant in the early eras): in non-spherically symmetric geometries in the Early Universe.

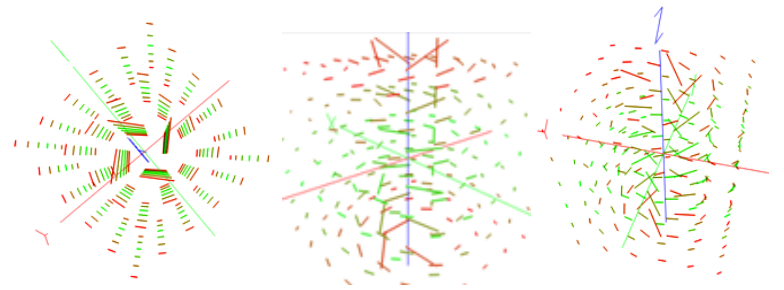

Fig.14. The induced torsion for non-spherically symmetric geometries by the curvature coupling to fermion spin. The attractor hole in the figure 13, can be constructed from the fermion couplings showed in these computational models.

Such geometries can be consigned sensing and perceiving through sensor whose $g$-cell include a hyper-sensible component that consists of a "sphere" composite of a nonNewtonian fluid (that perceive the Gaussian curvature units) which can be deformed by variations of gravity to start of the values of each parasite capacitance. The little alteration of their surface in non-harmonic "creases" produces an alteration of their charge $[12,23,24]$ (see the figure 14).

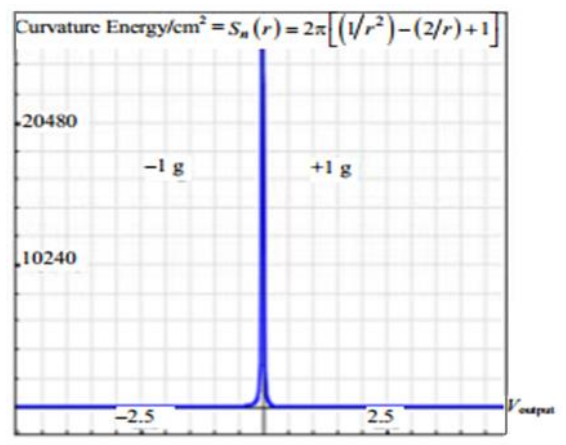

(a)

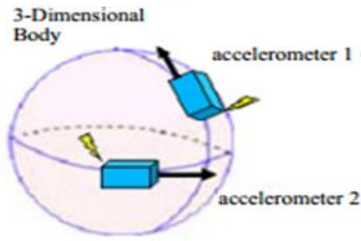

(b)

Fig.14. The curvature will be able to express itself like a Gaussian curvature according to spherical harmonics given by Legendre polynomials. 


\section{ACKNOWLEDGMENT}

I am very grateful with the organizers of Science and Information International Conference-2014, for their invitation to give a talk on my realized researches to design and development of a sensor of quantum gravity. The material of this paper is an extension of the presented work in London, UK.

\section{TECHNICAL NOTATION}

$K$ - Curvature as general concept of roundness property. Also used in the paper as Gaussian curvature in a point $p$

$k-$ Gaussian curvature along of the geodesic or surface $\Theta_{E}-$ Spherical operator which related with the spherical map. $k_{i}$ - Principal curvature in the principal ith-direction. In our research $\mathrm{i}=1,2$, only

$V-$ Voltage

$A-$ Area

$\mathcal{L}\left(H\left(\Omega^{2}(M)\right)\right)$ - Space of spectral transformations on curvature 2 - forms given in space $\Omega^{2}(M)$

$M$-Space whose curvature is measured. In our study $M$, represent 2 -dimensional surfaces or 3-dimensional bodies. $S^{2}-2$-Dimensinal sphere. Also is the 2-dimensional sphere used in the spherical map to design our curvature sensor

$r(P)$ - Evaluation of curvature radius from the product from their inverse principal curvatures.

$\Omega$ - Curvature form in the 4 , and 4 -dimensional spaces

$J_{1-r, p}-$ Bessel function of first specie

$Y_{1-r, 1-r}-$ Bessel function of second specie

SOIC - Small-outline integrated circuit

EDMC - Electromagnetic device to measure curvature

$m V-$ Micro-volts

$\left(Z^{\alpha}, W_{\alpha}\right)-$ Ambitwistor element whose elements are invariant-covariant fields

$S U(2)$ - Group that defines the finite actions through unitary anti-Hermitians matrix of range 2

ASIC - Application-specific integrated circuit

$Q E D$ - Quantum Electrodynamics

$Q F T$ - Quantum field theory

$S U S Y$ - Super-symmetry theory

$F R W$ - cohomology- Friedman-Robertson-Walker metrics. The cosmological principle (principle of homogeneity and isotropy of the universe to great scale). The cohomology are the relations of similarity in dual spaces.

\section{REFERENCES}

For papers published in translation journals, please give the English citation first, followed by the original foreign-language citation [6].
[1] Blumenhagen, Ralph; Lüst, Dieter; Theisen, Stefan (2012), Basic Concepts of String Theory, Theoretical and Mathematical Physics, Springer, p. 487, "Orbifolds can be viewed as singular limits of smooth Calabi-Yau manifolds".

[2] T. Rothman, S. Boughn, (2006). "Can Gravitons be Detected?". Foundations of Physics 36 (12): 1801-1825. arXiv:grqc/0601043

[3] N. E. Mavromatos, S. Sarkar, "CPT-Violating Leptogenesis induced by Gravitational Defects," High Energy Physics - Phenomenology KCLPH-TH/2012-23; LCTS/2012-28, pp1-22.

[4] J. Ellis, N. E. Mavromatos, S. Sarkar, "Environmental CPT Violation in an Expanding Universe in String Theory," High Energy Physics Phenomenology, KCL-PH-TH/2013-13, LCTS/2013-07, CERN-PHTH/2013-079, pp.1-8, arXiv: 1304.5433

[5] M. Kalb, P. Ramond (1974). "Classical direct interstring action". Phys. Rev. D 9 (8): 2273-2284.

[6] S-S. Chern, J. Simons (1974), Characteristic forms and geometric invariants, The Annals of Mathematics. Second Series 99 (1): 48-69,

[7] A. Boyarsky, A. Neronov, O. Ruchayskiy, M. Shaposhnikov , "Restrictions on parameters of sterile neutrino dark matter from observations of galaxy clusters," Physical Review D, 74 (10) 103506.

[8] Bulnes, F. "Electromagnetic Gauges and Maxwell Lagrangians Applied to the Determination of Curvature in the Space-Time and their Applications," Journal of Electromagnetic Analysis and Applications, Vol. 4 No. 6, 2012, pp. 252-266. doi: 10.4236/jemaa.2012.46035.

[9] Van Vliet L. J.; Verbeek, P.W. Curvature and bending energy in digitized 2D and 3D images, in: SCIA'93, Proc. 8th Scandinavian Conference on Image Analysis, Tromso, Norway, 1993, 1403-1410.

[10] Król, J. Quantum gravity insights from smooth 4-geometries on trivial R $^{4}$, Quantum Gravity, Intech. Chapter IV, pp53-78, Croatia, 2012.

[11] Bulnes, F.; Hernandez, E.; Maya, J. Design of Measurement and Detection Devices of Curvature Through of the Synergic Integral Operators of the Mechanics on Light Waves. Proceedings International Mechanics Engineering Conference and Exposition, Orlando Florida, USA, November 16; ASME, 2009, 91-102. http://dx.doi.org/10.1115/IMECE2009-10038.

[12] Bulnes, F. Martínez, I. Mendoza, A. and Landa, M. "Design and Development of an Electronic Sensor to Detect and Measure Curvature of Spaces Using Curvature Energy," Journal of Sensor Technology, Vol. 2 No. 3, 2012, pp. 116-126. doi: 10.4236/jst.2012.23017.

[13] Pallás, R. and Webster, J. G. Sensors and Signal conditioning 2nd ed. John Wiley \& Sons, USA, 2001.

[14] Nashelky B. Electronics: Circuit Theory and Electronic Devices, $1^{\text {st }}$ ed.; Pearson Prentice Hall, USA, 2003.

[15] Bulnes, F. Research on Curvature of Homogeneous Spaces, 1st ed.; TESCHA: State of Mexico, Mexico, 2010; pp. 44-66.

[16] A-Wollmann Kleinert and F. Bulnes, "Spinor Technology: the Field Formalism to the Duality between Quantum Particles and their Waves," Journal on Photonics and Spintronics, Vol. 2, n. 2, 2013, pp9-18.

[17] A. Wollman-Kleinert, F. Bulnes and D. Guderian, "Measurable Quantities of Quantum Field Theory: An Reviewing of Scalar Field Theory and their Applications," Journal on Photonics and Spintronics Vol. 3, no. 2, 2014, pp.10-19.

[18] Bulnes, F. Doctoral course of mathematical electrodynamics, Internal. Proceedings Appliedmath 2, SEPI-IPN and IM-UNAM, México City, Mexico, 2006, 398-447. ISBN: 970360358-0.

[19] Smoot Group, "The Cosmic Microwave Background Radiation," Lawrence Berkeley National Laboratory, Berckeley, 2008.

[20] Holmgren, O.; Kokkonen, K; Veijola, T.; Mattila, T.; Kaajakari, V.; Oja, A.; Knuuttila, J. V.; Kaivola, M. Analysis of vibration modes in a micromechanical square-plate resonator, Journal of Micromechanics and Microengineering, 2009, 19-1, p.015028.

[21] Kaajakari, V. Closed form expressions for RF MEMS switch actuation and release time, Electr. Let., 2009 (43)-3, pp. 149-150 DOI: 10.1049/el:20093281

[22] Volovik, G. E (1999). "Fermion zero modes on vortices in chiral superconductors". JETP Letters 70 (9): 609. 
(IJACSA) International Journal of Advanced Computer Science and Applications, Special Issue on Extended Papers from Science and Information Conference 2014

[23] Alastalo A. T.; Kaajakari, V.Intermodulation in capacitively coupled microelectromechanical filters, IEEE Electron Device Letters, 2005, 26-, pp.189-191.
[24] Lau, J.; Lee Ch.; Premachandran C.; Aibin, Y. Advanced MEMS Packaging (Electronic Enginnering, Kindle Editon, USA, 2010. 